Proc. Indian Acad. Sci. (Earth Planet. Sci.), Vol. 100, No. 2, June 1991, pp. 127-144. (C) Printed in India.

\title{
On the gross predictability of tropical monsoonal depressions
}

\author{
M SANKAR-RAO, PRASHANT GOSWAMI \\ and H V NAGARAJ UPADHYAYA \\ Department of Aerospace Engineering and Centre for Atmospheric Sciences, Indian Institute \\ of Science, Bangalore-560012, India \\ MS received 22 July 1990; revised 1 February 1991 \\ Abstract. The experiments reported here emphasize the importance of observations in the \\ prediction of tropical cyclones. Towards this end a symmetric numerical model in which \\ convection, parametrized by the Arakawa-Schubert (AS) scheme, was adopted. A mean \\ thermodynamical state, which represents the monsoon conditions over the Bay of Bengal, \\ with constant moist static energy for the mixed layer was adopted. Experiments were then \\ done with different initial conditions. \\ We found that tropical cyclone development measured by the central pressure was very \\ sensitive to the initial convergence field. In the present state of satellite technology, it was \\ impossible to predict even a gross parameter like the central pressure with an accuracy better \\ than $6 \mathrm{mb}$ for 12 hours. However, it was seen that under a variety of initial conditions the \\ final state characterized by the magnitude of the central low pressure remained practically \\ unaltered. We suggest that, given the necessary conditions for genesis, the final state of the \\ cyclone acts as an attractor (regarding its central pressure) and the diverse initial conditions, \\ under the influence of thermodynamic forcing, will lead to the same final state.
}

Keywords. Cyclones; prediction; convective parametrization; central pressure; physical model; numerical model.

\section{Introduction}

In the monsoon season the weather over India is influenced by tropical cyclones and depressions which generally drift over to the Bay of Bengal from the east. Occasionally these cyclones develop in situ over the Bay.

The mechanism for the growth of tropical cyclones is well established. The co-operation between a large scale low-pressure system and induced cumulus scales leads to intensification of a tropical cyclone. The convective mechanism induced by the large scales is known as conditional instability of the second kind (CISK). This concept was introduced by Ooyama (1964) and Charney and Eliassen (1964). Many essential features of tropical cyclones were simulated by numerical experimenters using different parameterization schemes for CISK. We refer to Ooyama (1969); Yamasaki (1968a,b,c, 1977a,b); Rosenthal (1970, 1971, 1973); Anthes et al (1971); Kurihara and Tuleya (1974); Kurihara (1975); Wada (1977, 1979); Emanuel (1986, 1989); Rotunno and Emanuel (1987); Jong-Jin-Baik et al (1990). Emanuel, in a series of papers, showed the importance of (a) heat transfer from the ocean (b) the finite amplitude of the initial perturbation and (c) cumulus downdrafts for development of tropical cyclones.

Wada's model, which is adopted here, is a symmetric model in which convection was parameterized by the Arakawa-Schubert (AS) scheme (Arakawa and Schubert 
1974). Wada simulated a realistic tropical cyclone with this model. Wada's model provides a good research tool for understanding tropical cyclones and depressions.

Some of the questions on Bay cyclones are:

(i) Why are Bay cyclones weaker than cyclones elsewhere? Is it because of the general structure of the basic state of the atmosphere and the sea, or is it because they spend lesser time over oceans?

(ii) As time progresses the cyclones form in the southern Bay. Is the shift in location due to shfits in the thermal and moisture structure, which is critical according to the earlier results, or is it because of shifts in horizontal shear zones that might be responsible for triggering an initial finite amplitude perturbation?

(iii) Both cold and warm core cyclones have been reported in the literature. How sensitive is the development of cyclones to such differences in the initial temperature structure?

An important requirement for answering such questions is observational data, which are sparse during the initial stages of cyclone formation.

We will focus attention on the last question of sensitivity which is linked with the initial perturbation. Conventional observations are generally not available over the seas in the initial stages. The satellites at present generally give the cloud cover and temperature structure. The accuracy of temperature measurements by satellites is at best $0.5^{\circ} \mathrm{C}$ at present. In such circumstances, one of the questions that arises is the following: Is it possible to say, with the help of satellite-derived temperatures and a model, whether a depression is likely to develop or not and what its intensity is likely to be in the final state? Thus the prediction question raised here is about some crucial 'gross parameters'. The interest here is not on the prediction of detailed spatial and temporal behaviour of the entire field as in the classical predictability studies but on certain dynamical parameters. Prediction of gross parameters is very useful sometimes. Suppose we can predict general circulation index. Such a prediction may not be able to tell us about the detailed structure of the global atmospheric fields but it can tell us whether we are likely to have high index or low index regime. We name such prediction as "gross prediction". The idea of gross prediction is therefore in a way akin to prediction of averages, here spatial averages, although the concept is more general.

That suitable averages may have longer ranges of predictability was expressed by earlier workers (Shukla 1981, 1985; Charney et al 1983). For example Shukla has shown that the monthly means of the dynamical variables had longer ranges of predictability. The parameters of gross prediction that we choose must obviously depend on the physical problem and experience. However, an element of choice or subjectivity in the choice of prediction variables is inherent in all prediction schemes (Shukla 1985).

To answer the above question we first simulated a tropical depression taking the mean state of the Bay of Bengal and a warm core initial perturbation. We treated this as the control run. The second experiment was with an initial 'warm core below and cold core above' with the same mean conditions. As we cannot use the gradient wind relation for the second type of temperature perturbations, we resort to dynamical initialization to find a balanced initial state. We also describe some experiments on dynamical initialization. In the other experiments, we perturb the initial structure of the control run by superposing random perturbatons of different magnitudes; and 
compare the development with the control run. Before describing the results of the experiments, we, briefly present the details of the model.

\section{Physical and numerical model}

This model is symmetric. The basic equations in the cylindrical coordinates are the following:

$$
\begin{aligned}
\partial v_{r} / \partial t= & -\left(v_{r} \partial v_{r} / \partial r+w \partial v_{r} / \partial z\right)+\left(f+v_{\theta} / r\right) v_{\theta}-\theta \partial \phi / \partial r \\
& +K_{r}\left(\nabla_{1}^{2}-1 / r^{2}\right) v_{r}+1 / \bar{\rho} \partial \tau_{r} / \partial z \\
\partial v_{\theta} / \partial t= & -\left(v_{r} \partial v_{\theta} / \partial r+w \partial v_{\theta} / \partial z+\left(f+v_{\theta} / r\right) v_{r}+K_{F}\left(\nabla_{i^{-}}^{2} 1 / r^{2}\right) v_{\theta}\right. \\
& +1 / \bar{\rho} \partial \tau_{\theta} / \partial z, \\
\partial \phi / \partial z= & -g / \theta \\
\frac{q}{\partial z} \bar{\rho} w= & \frac{1}{r} \frac{d}{\partial r}\left(\bar{\rho} v_{\theta} r\right) \\
\partial \theta / \partial t= & -\left(v_{r} \partial \theta / \partial r+w \partial \theta / \partial z\right)+1 / \bar{\rho} \phi\left[M_{c} \partial s / \partial z+D\left(S_{c}-S-L \hat{l}_{c}\right)\right] \\
& +L C / \phi+K_{\theta} \nabla_{1}^{2} \theta+F_{S} /\left(C_{P} \bar{\phi}\right), \\
\partial \chi / \partial t= & -\left(v_{r} \partial \chi / \partial r+w \partial \chi / \partial z\right)+1 / \bar{\rho}\left[M_{\mathrm{c}} \partial \chi / \partial z D\left(\hat{\chi}_{c}-\chi+\hat{l}_{c}\right)\right] \\
& -C+K_{\chi} \nabla_{1}^{2} \chi+F_{\chi} / L,
\end{aligned}
$$

where

$$
\phi=C_{P}\left(P / P_{0}\right)^{\kappa}
$$

The notations are given in appendix 2. The overbar denotes the functions of height only, subscript $c$ denotes the values inside clouds and the carat $\left({ }^{\wedge}\right)$ denotes the values at the cloud top.

For frictional stress and energy and moisture fluxes the aerodynamic bulk formulae were used as follows:

$$
\begin{aligned}
& \tau_{r}=\bar{\rho} K_{v} \partial v_{r} / \partial z, \\
& \tau_{\theta}=\bar{\rho} K_{v} \partial v_{\theta} / \partial z, \\
& \tau_{r s}=\bar{\rho}_{s} C_{D}\left|V_{S}\right| v_{r s}, \\
& \tau_{\theta s}=\bar{\rho}_{S} C_{D}\left|V_{S}\right| v_{\theta S}, \\
& F_{S}=C_{P} C_{D}\left|V_{S}\right|\left(T_{S}-T_{M}\right) / Z_{B}, \\
& F_{x}=L C_{D}\left|V_{S}\right|\left(\chi_{S}-\chi_{M}\right) / Z_{B} .
\end{aligned}
$$

The suffix $S$ denotes surface values and the suffix $M$ denotes mixed layer values. $Z_{B}$ is the height of the mixed layer. Note that the continuity equation (4) is the anelastic equation.

The vertical structure of the finite difference model, given in figure 1 , consists of a thin mixed layer denoted by subscript $\boldsymbol{B}$ and five levels above it. The model atmosphere is divided into five layers. Each layer is denoted by half integers, and is 


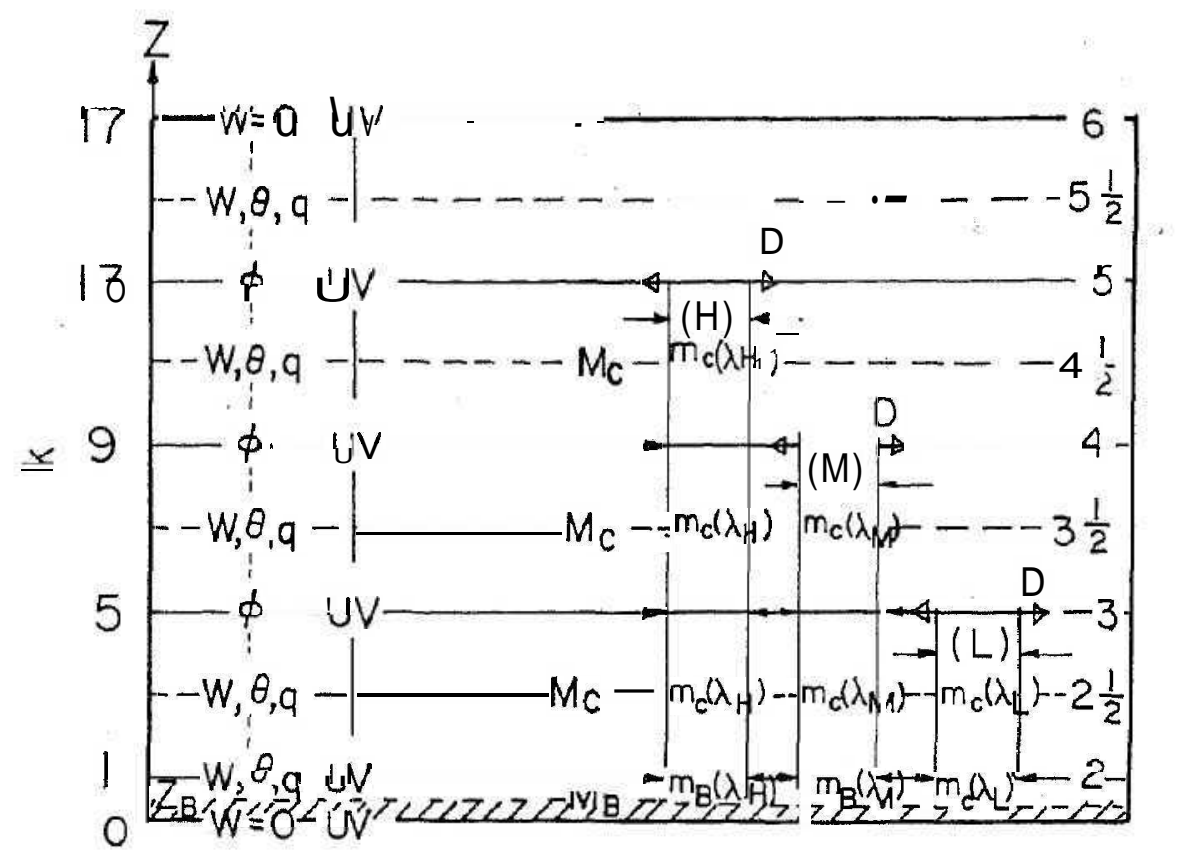

Figure 1. Vertical structure of the model.

bounded by integer levels $v_{r}, v_{\theta}$ and $\phi$ are defined at the integer levels, while $W, 9$ and $\chi$ are defined at half integer levels.

The time variation of the moist static energy in the mixed layer $h_{M}$ is approximately given by

$$
\partial h_{M} / \partial t=-v_{r} \partial h_{M} / \partial r-K_{\theta} \nabla_{1}^{2} h_{M}+F_{H},
$$

where $F_{H}$ is given by

$$
F_{H}=C_{D}\left|V_{S}\right|\left(h_{S}-h_{M}\right) / Z_{B}
$$

In the present experiments, $h_{M}$ was kept constant. The boundary conditions at the top and bottom were

$$
W=0 \text { at } z=0 \text { and } z=Z_{T}
$$

This condition along with (4) filters external gravity waves. However, it may cause reflection of vertically propagating waves. It will be interesting to study the effect of other boundary conditions, like open boundary conditions, in future.

The lateral boundary conditions are

$$
\begin{aligned}
& v_{r}=v_{\theta}=0 \text { at } r=0 \text { and } r=r_{\max }, \\
& \partial \phi / \partial r=\partial \theta / \partial r=d x / 8 r=0 \text { at } r=0 \text { and } r=r_{\max },
\end{aligned}
$$

$r_{\max }$ is taken to be $2500 \mathrm{~km}$ to reduce the influence of lateral boundaries (table 1).

A staggered forward time differencing scheme was used. Upwind differencing was used for space differencing. The grid size in the horizontal direction was variable getting smaller towards the centre. Twentyone grid points were used. The grid size was approximately $20 \mathrm{~km}$ near the centre, while at $r_{\max }$, the grid size was about $450 \mathrm{~km}$. Such a staggered grid can create numerical problems of reflection if the staggering 
Table 1. Grid positions of the rings.

\begin{tabular}{|c|c|c|c|c|c|}
\hline$J$ & $\begin{array}{c}r \\
\text { (in km) }\end{array}$ & $\mathrm{J}$ & $\begin{array}{c}r \\
\text { (in km) }\end{array}$ & $J$ & $\begin{array}{c}r \\
\text { (in km) }\end{array}$ \\
\hline 1 & 20 & 8 & 198 & 15 & 787 \\
\hline 2 & 40 & 9 & 244 & 16 & 951 \\
\hline 3 & 60 & 10 & 298 & 17 & 1147 \\
\hline 4 & 80 & 11 & 364 & 18 & 1382 \\
\hline 5 & 102 & 12 & 443 & 19 & 1665 \\
\hline 6 & 128 & 13 & 538 & 20 & 2004 \\
\hline 7 & 160 & 14 & 651 & 21 & 2500 \\
\hline
\end{tabular}

was not gradual. However, the results obtained with this grid were realistic and acceptable.

For convenience in computation the scalar fields of 6 and $\phi$ were divided into two parts: an undisturbed value and a perturbed value. The hydrostatic equation was written separately as follows:

$$
\begin{aligned}
& e(r, z, t)=\bar{\theta}(z)+\theta^{\prime}(r, z, t), \\
& \phi(r, z, t)=\bar{\phi}(z)+\phi^{\prime}(r, z, t), \\
& \partial \bar{\phi} / \partial z=-g / \bar{\theta}, \\
& \partial \phi^{\prime} / \partial z=g / \bar{\theta}\left[\theta^{\prime} / \bar{\theta}-\left(\theta^{\prime} / \bar{\theta}\right)^{2}\right]
\end{aligned}
$$

Initially $\theta^{\prime}$ is assumed to have the form

$$
\&(r, z)=0 \cdot 20\left\{\cos \left(\pi r / r_{0}\right)+1\right\} \sin \left(\pi z / Z_{T}\right) W_{T}(z) .
$$

Thus the temperature perturbation vanished beyond $r=r_{0} . r_{0}$ is taken as $200 \mathrm{~km}$. $\theta^{\prime}(r, z)$ was a maximum at $r-0$ and $\mathrm{z}=Z_{\mathrm{T}} / 2$ and decreased with $r$, if the weighting factor $W_{T}(z)$ was unity.

The initial fields were calculated by first computing $\phi^{\prime}$ using (21), with $\phi^{\prime}=0$ at the top. With this $\phi^{\prime}$ field the initial tangential wind field was computed utilizing the gradient wind equation given by

$$
v_{\theta}=\left(-f r+r\left(f^{2}+4 \bar{\theta} / r \cdot \partial \phi^{\prime} / \partial r\right)^{1 / 2}\right) / 2 .
$$

For $v_{\theta}$ to be real we must have

$$
f^{2}>-4 \bar{\theta} / r \partial \phi^{\prime} / \partial r \text {. }
$$

For $\partial \phi^{\prime} / \partial r<0$ (for highs) there is a possibility of (24) being violated. In that case, dynamical initialization was adopted.

In this model, large scale condensation occurs when the air is supersaturated. The water vapour available above the saturation value is condensed.

The convective heating in this model was parameterized by AS parameterization. The cloud base level $Z_{c}$ was taken as the condensation level. Instead of an entrainment rate $A$, the detrainment level $\boldsymbol{Z}_{D}(\lambda)$ was used as the independent variable in this model. 
The important assumptions in Wada's model are the following:

$$
\begin{aligned}
& h\left(Z_{1}+1 / 2\right) \leqslant h_{M} \leqslant h^{*}\left(Z_{1}+1 / 2\right), \\
& T_{(1+1 / 2)}=\text { constant. }
\end{aligned}
$$

The restriction on $h_{M}$ by (25) prevents uninhibited development of the cyclone. (AS) parameterization was slightly simplified by Wada in this model. The (AS) parameterization and its simplification are given in appendix 1 . The results of the experiment are next described.

\section{Results of numerical experiments}

\subsection{Background state}

The background state representing the climatological (20-year mean) mean vertical structure for Calcutta for June is shown in figure 2. These data were published by the India Meteorological Department. The curves were representative of a tropical moist-atmosphere. The mixed layer moist static energy $h_{M}$ was $85.66 \mathrm{cal} / \mathrm{g}$. This was considerably larger than what Wada used $(80 \mathrm{cal} / \mathrm{g})$.

The ambient surface pressure was taken to be $1000 \mathrm{mb}$. We have also taken $h_{M}=$ constant $=h_{B}$, and $\delta_{1}=\delta_{2}=0$ in all the experiments.

\subsection{Experiment 1}

In this experiment the initial vortex structure was given by (22) with all weights $W_{T}(z)$ as unity. The initial temperature structure (figure 3) gives the highest potential

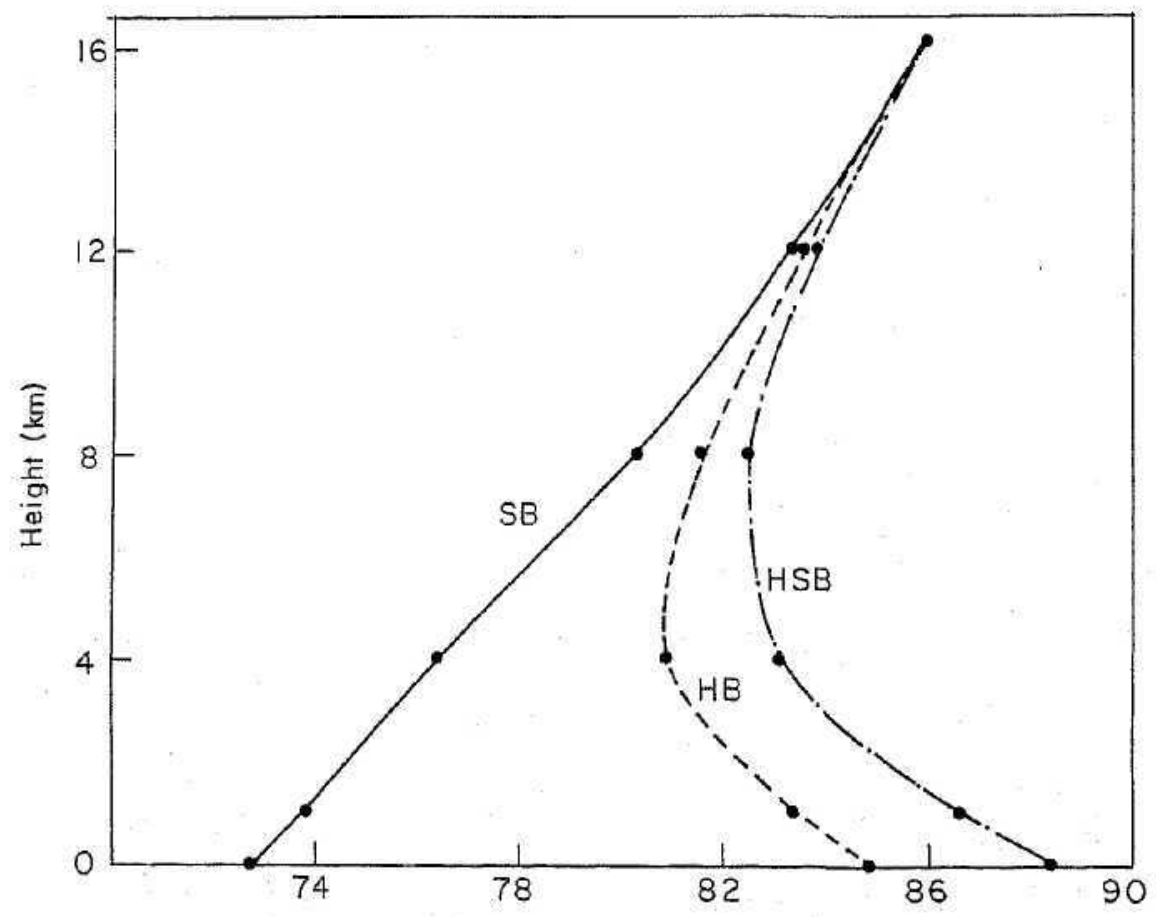

Figure 2. Static energy of the basic state in $\mathrm{cal} / \mathrm{g} ; \mathbf{S B}=$ dry static, $\mathrm{HB}=$ moist static, $\mathrm{HSB}=$ moist saturated static. 20-year mean for June, for Calcutta. 


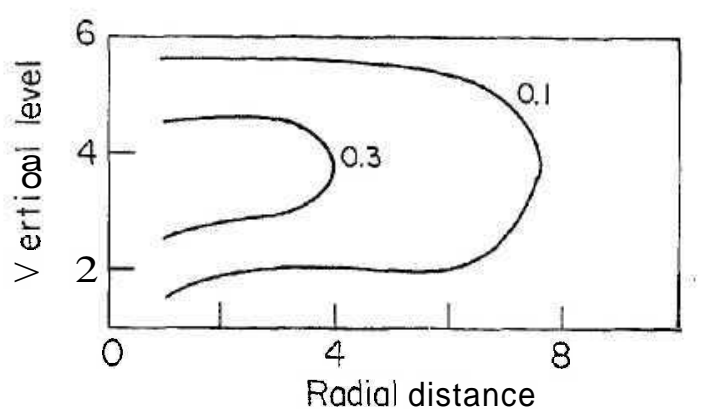

Figure 3. Temperature structure at time step $0 \mathrm{~h}$, expt 1 , in ${ }^{\circ} \mathrm{C}$.
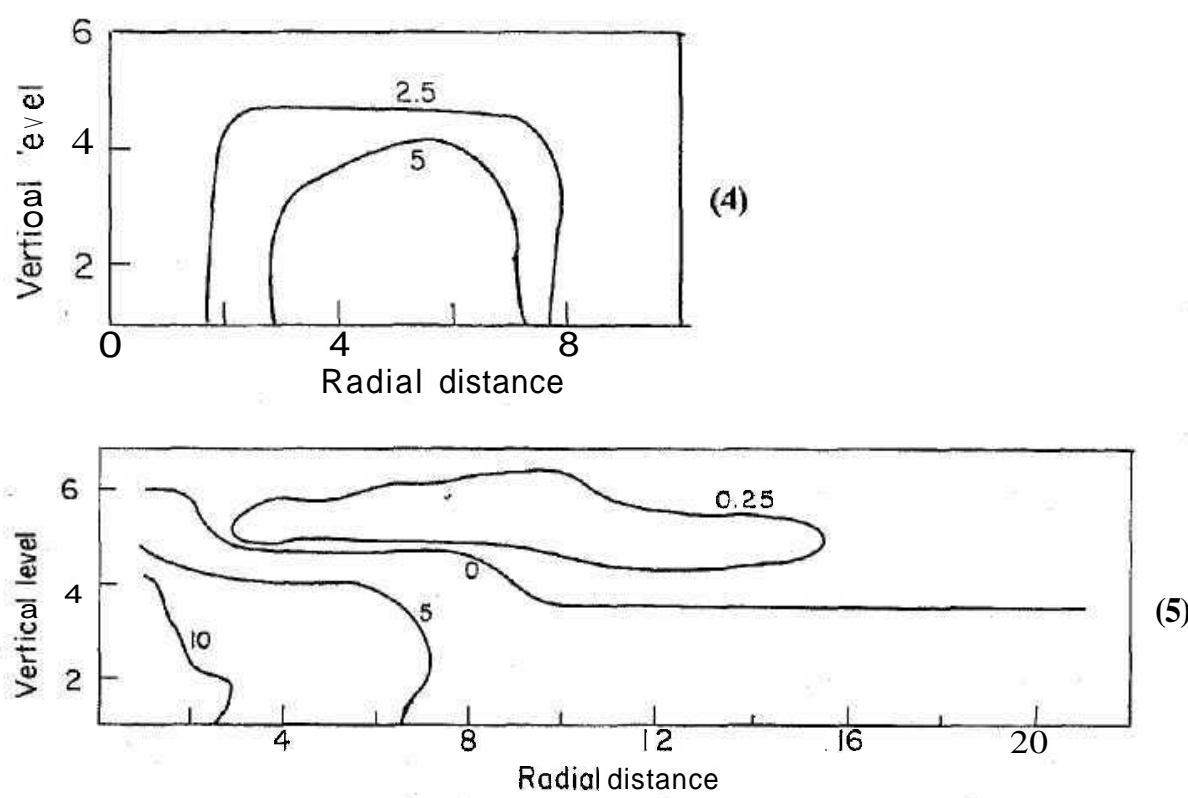

Figures 4,5. Tangential wind structure at time steps $0 \mathrm{~h}$ and $24 \mathrm{~h}$ respectively, expt. 1 , in $\mathrm{m} / \mathrm{s}$.

temperature perturbation of $04^{\circ}$ at level 4 at the central grid point decreasing to $0^{\circ}$ at $r-200 \mathrm{~km}$. Due to this warm temperature perturbation throughout the depth of the atmosphere (deep warm core initial structure) a weak low pressure was formed at the centre. The corresponding initial wind structure may be computed by the gradient wind relation. This is shown in figure 4 . The initial radial wind was considered to be zero in this type of static initialization. In dynamical initialization, there could be radial winds initially because of nonlinearities. The gradient wind initial conditions provide a cyclonic circulation around this low with the highest tangential winds of about $7.4 \mathrm{~m} / \mathrm{s}$ at levels 2 and 1 at about $128 \mathrm{~km}$ from the centre. With these initial conditions this experiment was run for 120 hours. The results are shown in figures 5 to 15 . We shall describe these results for the different parameters.

\subsection{Tangential winds (figures 4 and 5)}

The cyclone began to deepen almost immediately without any predevelopment stage. Within 48 hours strong cyclonic winds of about $19 \mathrm{~m} / \mathrm{s}$ were obtained in the lower layers at $r=80 \mathrm{~km}$. Thus, as the cyclone intensified the core of the maximum tangential wind shifted inwards. At the same time, strong anticyclonic winds of about $12 \mathrm{~m} / \mathrm{s}$ 

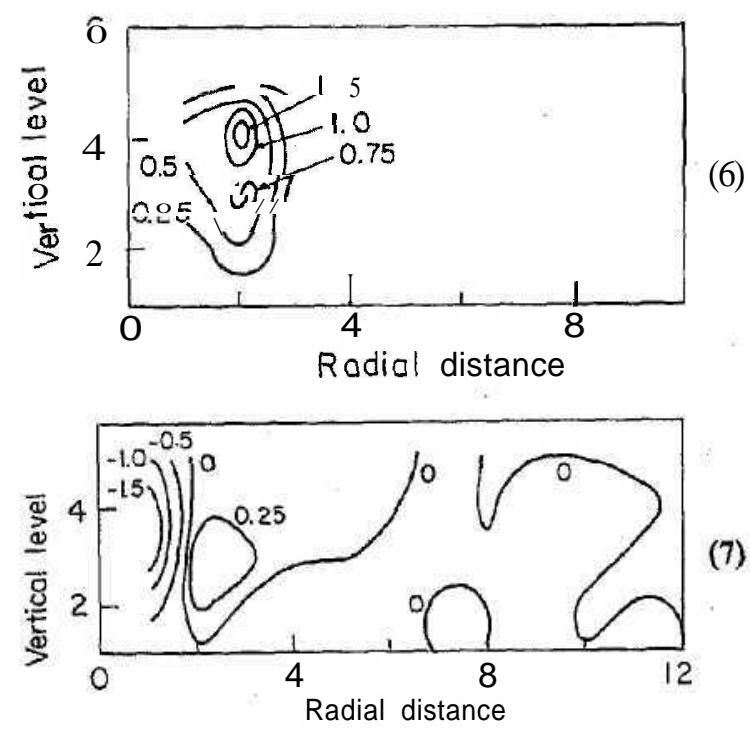

Figures 6, 7. Vertical wind structures at time steps $24 \mathrm{~h}$ and $48 \mathrm{~h}$ respectively, expt. 1 , in $\mathrm{m} / \mathrm{s}$.

were obtained at level 5 at $r=787 \mathrm{~km}$. Thus, the lower level low and upper level high were formed at this mature stage. After 48 hours the cyclone remained almost in a steady state and slightly decreased in intensity.

\subsection{Vertical wind (figures 6 and 7)}

At the mature state of $48 \mathrm{~h}$ strong sinking motion $(-1.5 \mathrm{~m} / \mathrm{s})$ at the centre and upward motion $(0.25 \mathrm{~m} / \mathrm{s})$ at $r=40 \mathrm{~km}$ with outward flow was noticed. Downward motion at the centre may be interpreted as an eye formation. After $48 \mathrm{~h}$ the subsidence at the centre disappeared. At $72 \mathrm{~h}$ the strongest upward motion was still near the central core (figure not shown). The coarse $(20 \mathrm{~km})$ resolution of the model at the centre did not allow one to discuss the eye formation. Away from the centre, cellular structure in the horizontal, with 3 week cells could be seen. At $96 \mathrm{~h}$ and $120 \mathrm{~h}$ the cyclone showed a steady state with slight weakening.

\subsection{Temperature (figures 8 to 10 )}

For these figures we see the development to the mature stage by $48 \mathrm{~h}$. At $48 \mathrm{~h}$ a central warm core with a temperature perturbation of about $+5^{\circ} \mathrm{C}$ formed. Almost the entire lower region of the cycione up to level 5, which corresponds to $13 \mathrm{~km}$ in height, was warmer than the environment. Above level 5 it was colder. Here the negative temperature perturbations were about $-1^{\circ}$. The cyclone was warmest at $72 \mathrm{~h}$, that is $24 \mathrm{~h}$ after the onset of the central downdraft. At $72 \mathrm{~h}$ the maximum positive perturbation temperature was about $7^{\circ} \mathrm{C}$ and was at level 4 in the central core. After $72 \mathrm{~h}$ the cyclone maintained the same structure but slightly weakened.

\subsection{Central pressure (figure 11)}

This figure shows that the cyclone was most intense at $42 \mathrm{~h}$. The fall of pressure at the centre was about $19 \mathrm{mb}$ in $42 \mathrm{~h}$. 

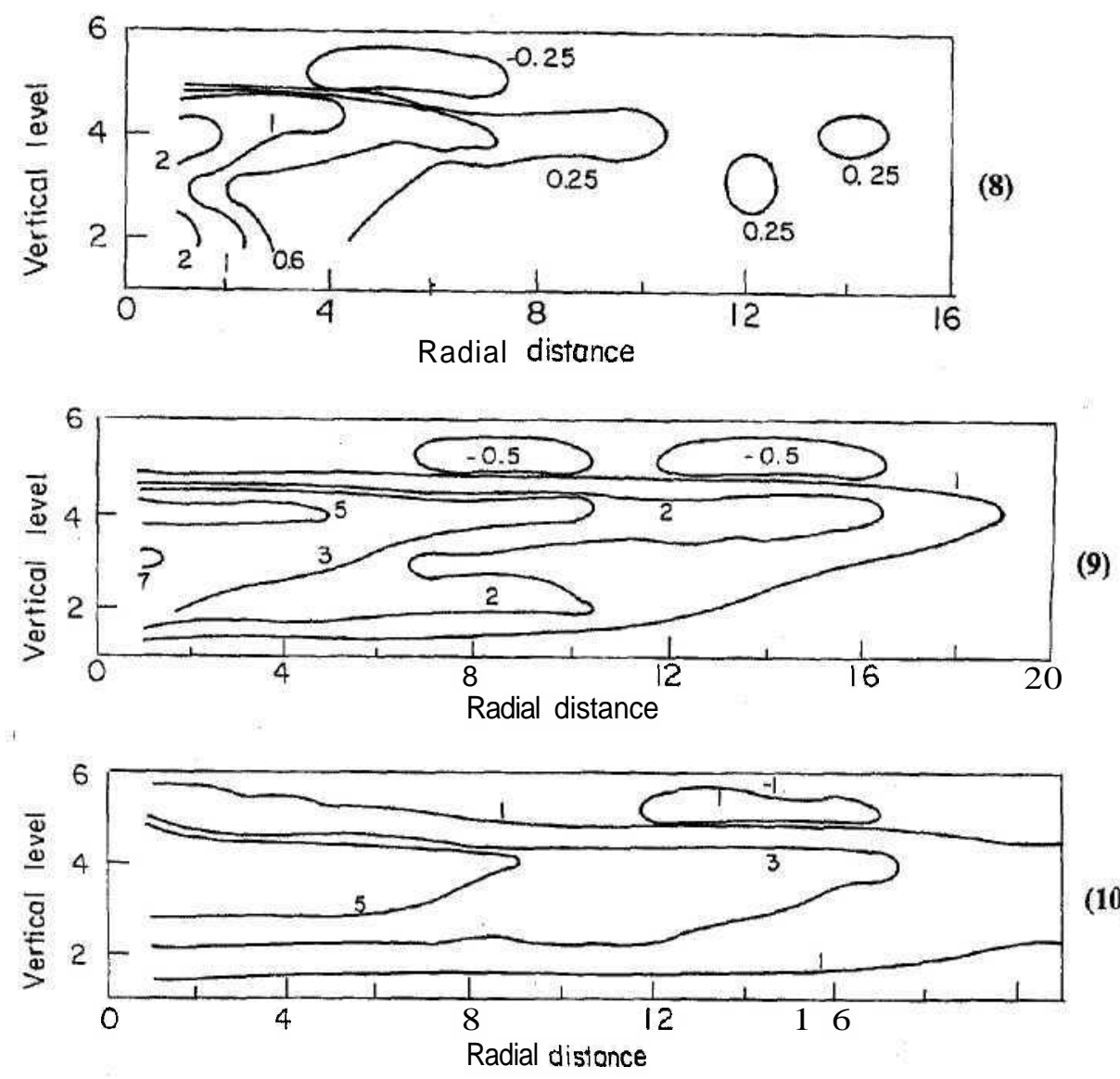

Figures 8,9,10. Temperature structure at $24 \mathrm{~h}, 48 \mathrm{~h}$ and $96 \mathrm{~h}$ respectively, expt. 1 , in ${ }^{\circ} \mathrm{C}$.

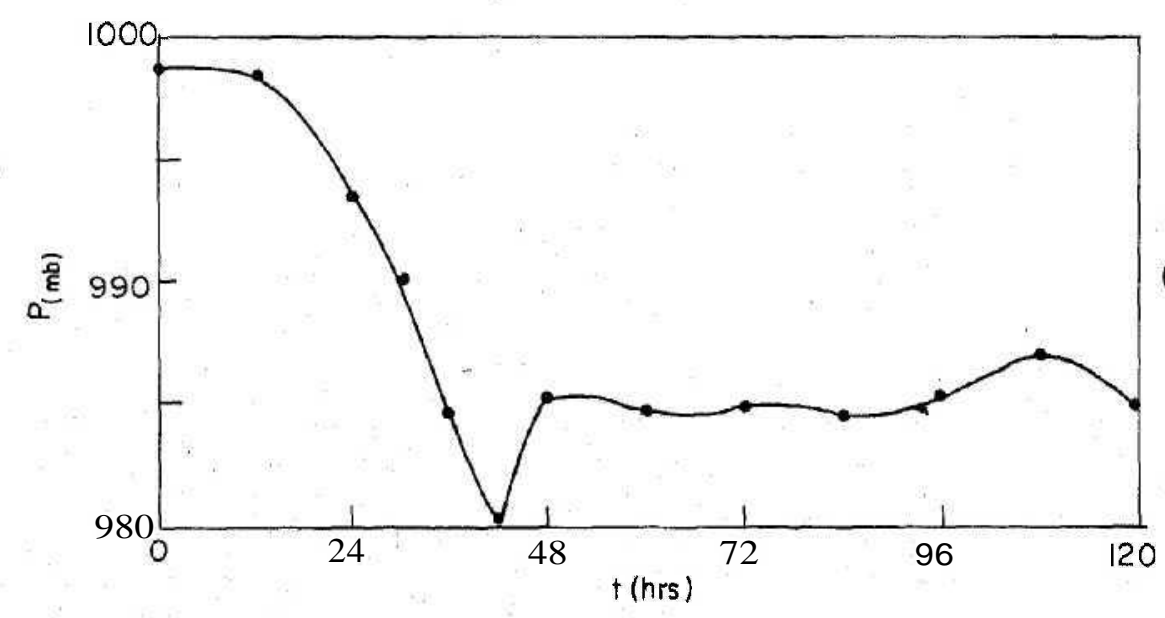

Figure 11. Central pressure $v s$ time, expt. 1.

\subsection{Cloud mass flux (figure 12)}

One can see in this figure large high cloud mass flux at the central core (2nd grid point $r=20 \mathrm{~km}$ ) at $24 \mathrm{~h}$. The value in the experiment was $352 \mathrm{~g} \mathrm{~m}^{-2} \mathrm{~s}^{-1}$. No medium or low clouds were produced at this stage. At $48 \mathrm{~h}$ high and medium cloud maxima were situated away from the centre, at $r=300 \mathrm{~km}$. In the central region, low clouds were formed. After $48 \mathrm{~h}$ only high clouds were of any significance. By $96 \mathrm{~h}$ the cyclone was almost cloud-free. 


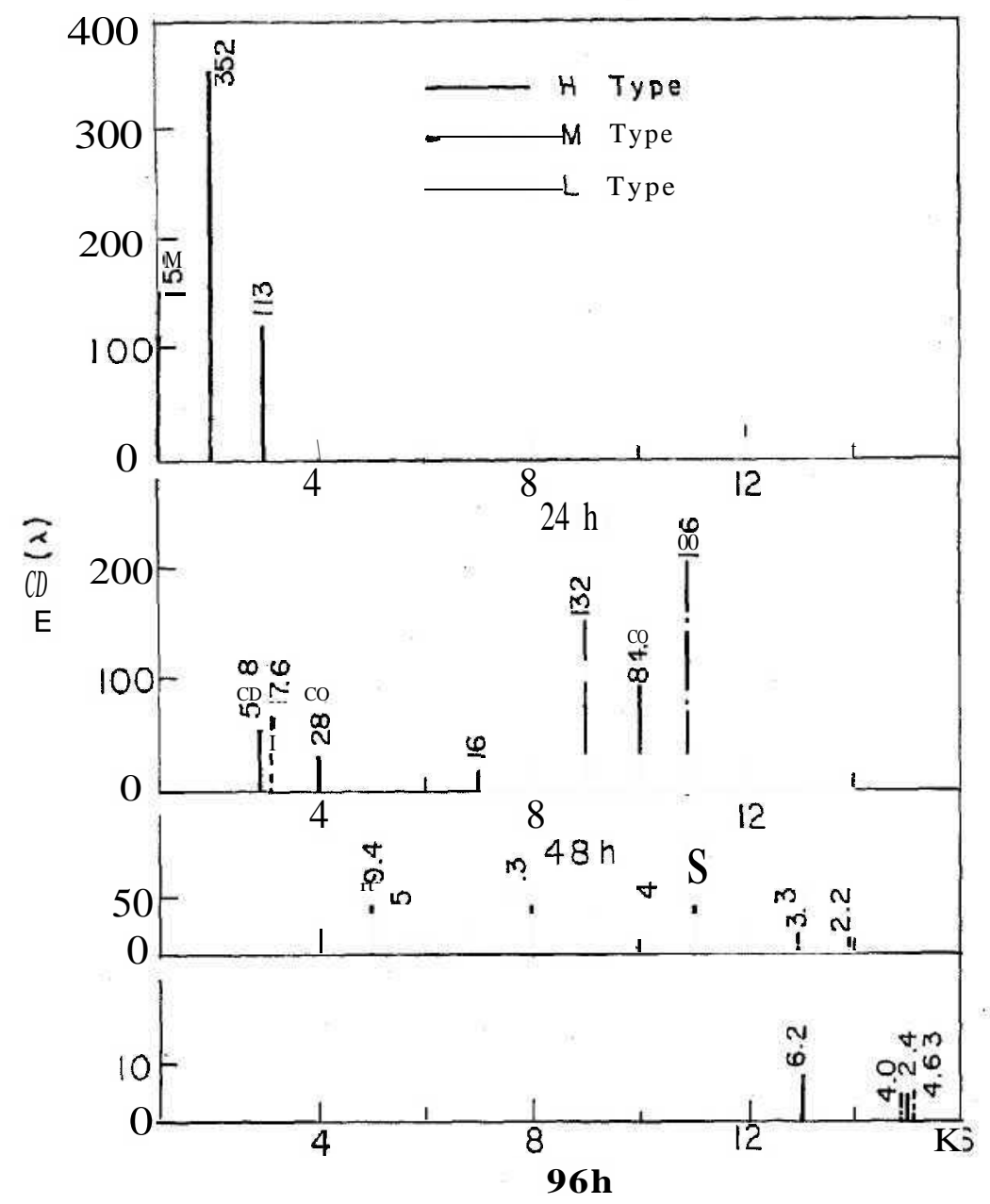

Figure 12. Cloud mass flux, expt. 1 , in $\mathbf{g ~ m}^{-2} \mathbf{s}^{-1}$.

In view of the large value of $h_{M}(=85.66 \mathrm{cal} / \mathrm{g})$ compared to that used for other parts of the tropics it was not surprising that the cyclone developed much faster in $48 \mathrm{~h}$ compared to $96 \mathrm{~h}$ in Wada's control run. However, the central pressure anomaly was just about $19 \mathrm{mb}$, while Wada found it to be about $25 \mathrm{mb}$. The main difference between the control experiment of Wada and this experiment was regarding $h_{M}$, which was kept constant here. This could be one of the reasons for a lesser fall of central pressure in this experiment, in view of the sensitivity of development to $h_{\boldsymbol{M}}$.

\section{Experiments la and $\mathbf{1 b}$}

In the above control experiment, a gradient wind balance was assumed initially. But this was only a special kind of balance. It was interesting to find out what happened if the initial balance was obtained differently, say, by dynamical initialization leading to a different set of initial conditions. Consequently, a series of experiments were performed to assess the impact of dynamical initialization. In la the initial state was at rest, while in $1 \mathrm{~b}$ the initial tangential wind field was the gradient wind as in the control experiment (Expt 1). Keeping the same fixed initial temperature structure in the control experiment, and by switching off the moisture equation, the model was 


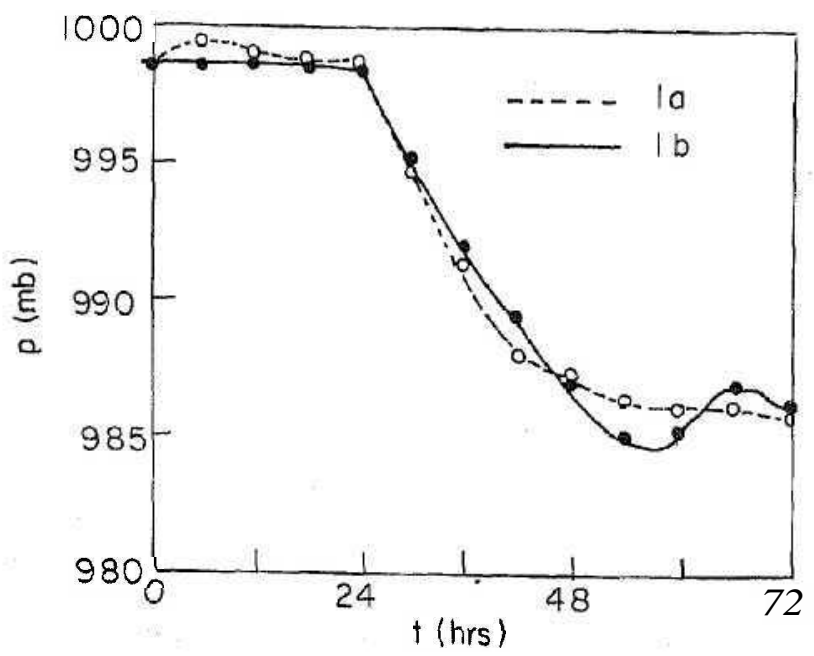

Figure 13. Central pressure $v$ s time, expt. la $(u=0)$ and $1 \mathrm{~b}(u \neq 0)$, dynamically initialized up to $24 \mathrm{~h}$ with different initial conditions.

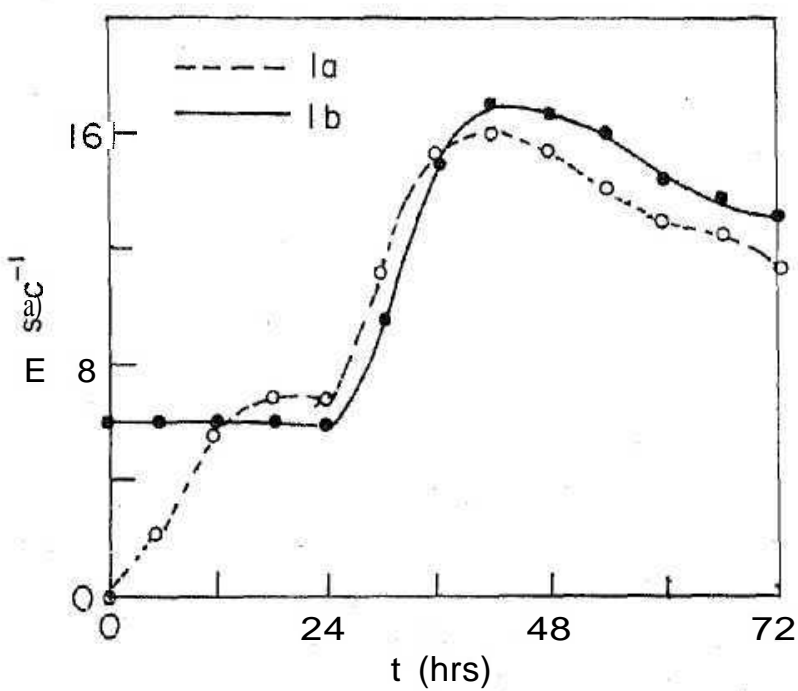

Figure 14. Tangential wind (at level 3 and $J=4) v s$ time, expt. la $(u=0)$ and $1 \mathrm{~b}(u \neq 0)$, dynamically initialized up to $24 \mathrm{~h}$ with different initial conditions.

integrated forward, with friction for $24 \mathrm{~h}$. This initialization produced radial winds. After obtaining the balanced initial fields, the model was integrated with the total physics of the model for another three days. In figures 13 and 14 we show the results on central pressure and tangential winds. We notice that dynamical initialization did not change the initial balanced gradient wind in Expt 1b. In Expt la, in which we started with no winds, the tangential wind field was close to the tangential gradient wind field, after 24 hours of adjustment. It may be seen that the evolution of the central pressure and the tangential winds were similar in both cases. When compared with the control experiment the development was faster. In the control experiment it took 40 hours for the central pressure to reach its minimum after the start, while in these experiments it took only 30 hours. This indicated the sensitivity of cyclone development to the initial radial winds. However, the final central pressure was nearly the same as in the control experiment. 

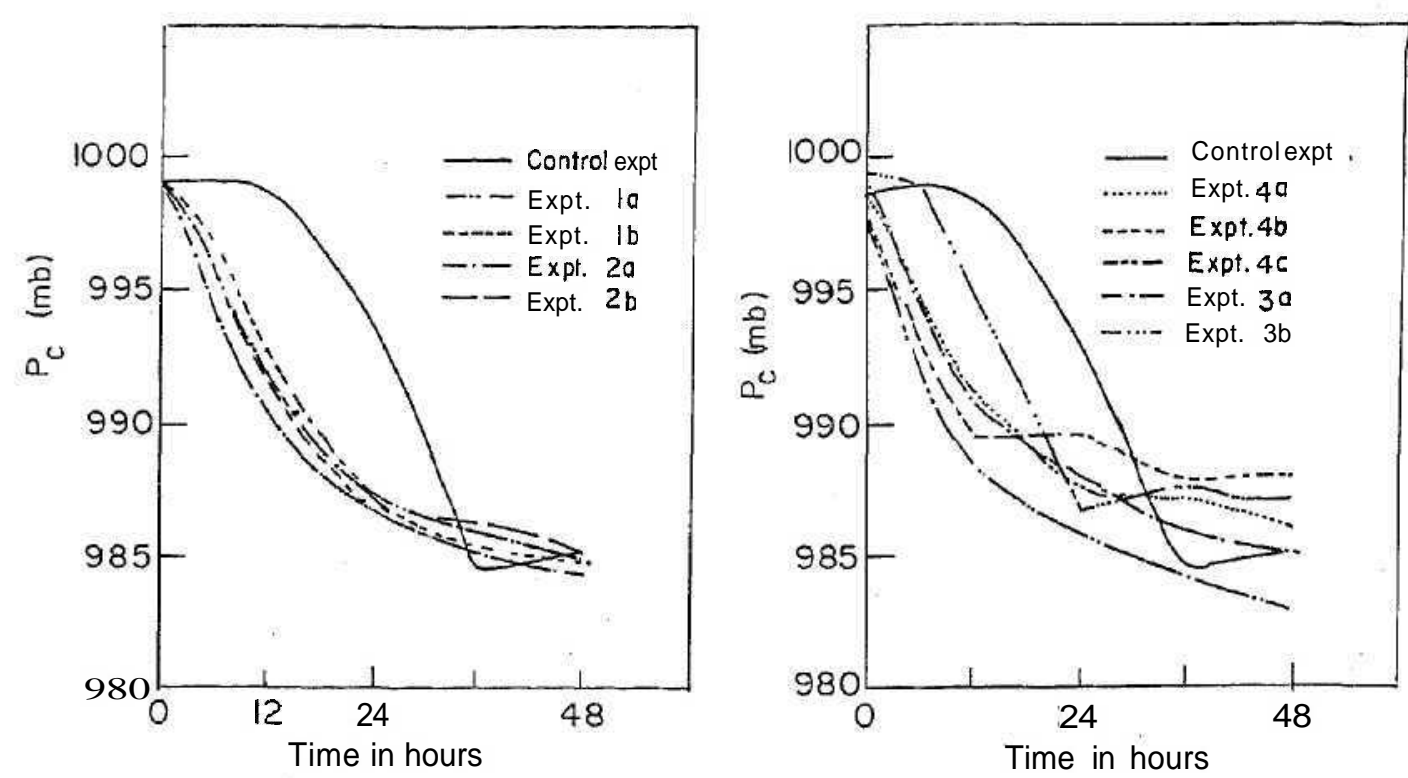

Figure 15. Central pressure history for different experiments.

\section{Experiments 2a and 2b. (figure 15)}

What happens if we extend the dynamical initialization up to $48 \mathrm{~h}$ instead of $24 \mathrm{~h}$ ? Two experiments which were performed to answer this question gave results which were close to each other. They were also similar to the results of experiments la and 1b. The steady-state central pressure was nearly equal to the one obtained in Expt 1 , la and $1 \mathrm{~b}$. Thus, experiments $\mathrm{la}, 1 \mathrm{~b}, 2 \mathrm{a}$ and $2 \mathrm{~b}$ were very close to each other indicating that their initial conditions were also very similar as seen in table $2 . \Delta u$ and $\Delta v$ for these experiments was about $0.2 \mathrm{~m} / \mathrm{s}$.

In these experiments there was no predevelopment stage. This could be due to the high amount of mixed layer moist static energy in these regions.

The experiments described so far were performed with a warm core initial perturbation. The following experiments were performed, where there was a warm core below and cold core above.

\section{Experiments 3a and 3b (figure 15)}

In these experiments we adjusted the weights to $(-1,-1,-1,+1,+1,+1)$, so that we have initially a cold pool above and a warm pool below. This produced a central high pressure throughout. The pressure gradient was directed away from the centre and reached a maximum at level 4 . Everything else was kept the same. We performed dynamic initialization for $48 \mathrm{~h}$. In experiment $3 \mathrm{a}(u=0)$, the initial state was one of no motion. In experiment $3 \mathrm{~b}(u \neq 0)$ initially, the gradient wind was obtained wherever possible keeping the motion at rest elsewhere. After $48 \mathrm{~h}$ of initialization the tangential winds in both cases were close to each other. There was no predevelopment stage even in these experiments. The steady-state central pressure was about $985 \mathrm{mb}$. Comparing the results of $3 \mathrm{a}$ and $3 \mathrm{~b}$ with $2 \mathrm{a}$, we noticed that the differences after $12 \mathrm{~h}$ were of the order of $1.5 \mathrm{mb}$ and remained so till the end of the experiments (figure 15 and table 2). 
Table 2. Initial average deviations from the control experiment $\overline{\Delta u}, \overline{\Delta v}$ (in m/s) AT(in ${ }^{\circ} \mathrm{C}$ ) and central pressure Ap (in mb) for different experiments. The central pressures $P_{\mathrm{c}}$ in $\mathrm{mb}$ for experiment 1 (control run) are given in the last row.

\begin{tabular}{|c|c|c|c|c|c|c|c|c|c|}
\hline \multirow[b]{3}{*}{ Expt } & \multicolumn{8}{|c|}{ Time in hours } & \multirow[b]{3}{*}{ Remarks } \\
\hline & 0 & 0 & 0 & 0 & 12 & 24 & 36 & 48 & \\
\hline & $\overline{\Delta u}$ & $\overline{\Delta v}$ & $\overline{A T}$ & Ap & Ap & Ap & Ap & Ap & \\
\hline la & 0.138 & 0.189 & 0.000 & $0 \cdot 100$ & $-7 \cdot 100$ & $-6 \cdot 200$ & 1.600 & 0.800 & $\boldsymbol{v}=0 ; \quad \mathrm{DI}=24 \mathrm{~h}$ \\
\hline $1 \mathrm{~b}$ & $0 \cdot 116$ & $0 \cdot 106$ & 0.000 & $0 \cdot 100$ & $-6 \cdot 500$ & $-6 \cdot 500$ & 0.800 & $1 \cdot 100$ & $D=v_{g} \quad \mathrm{DI}=24 \mathrm{~h}$ \\
\hline $2 \mathrm{a}$ & $0 \cdot 193$ & 0.205 & 0.000 & 0.200 & $-8 \cdot 200$ & $-7 \cdot 700$ & $1 \cdot 700$ & $1 \cdot 200$ & $v=0 \quad \mathrm{DI}=48 \mathrm{~h}$ \\
\hline $2 b$ & 0.086 & 0.082 & $0 \cdot 000$ & $0 \cdot 100$ & -6.800 & -6.600 & $1 \cdot 500$ & 0.400 & $v=v_{g} \quad \mathrm{DI}=48 \mathrm{~h}$ \\
\hline $4 \mathrm{a}$ & $0 \cdot 144$ & 0.231 & 0.006 & -0.000 & $-7 \cdot 300$ & $-6 \cdot 300$ & $2 \cdot 500$ & 1.000 & $v=0^{*} \quad \mathrm{DI}=48 \mathrm{~h}$ \\
\hline $4 b$ & 0.400 & 0.460 & 0.023 & -0.700 & $-9 \cdot 200$ & $-4 \cdot 000$ & $3 \cdot 300$ & $2 \cdot 900$ & $v=0 \quad \mathrm{DI}=48 \mathrm{~h}$ \\
\hline $4 c$ & 0.364 & 0.505 & 0.023 & -0.600 & $-10 \cdot 400$ & -7.800 & -0.500 & $-2 \cdot 100$ & $v=0 \quad \mathrm{DI}=48 \mathrm{~h}$ \\
\hline $3 a$ & $0-085$ & 0.410 & $0 \cdot 040$ & 0.600 & -7.900 & $-5 \cdot 500$ & $1 \cdot 300$ & -0.100 & $v=0 \quad \mathrm{DI}=48 \mathrm{~h}$ \\
\hline $3 b$ & $0 \cdot 127$ & 0.440 & 0.040 & 0.700 & $-0 \cdot 200$ & $-6 \cdot 900$ & -3.000 & 1.800 & $v=v_{6}, \quad \mathrm{DI}=48 \mathrm{~h}$ \\
\hline mean & $0 \cdot 183$ & $0 \cdot 292$ & 0.014 & 0.055 & -7.022 & -6.388 & 1.688 & 0.777 & \\
\hline$f f$ & $0 \cdot 117$ & $0 \cdot 162$ & 0.014 & 0.467 & 2.977 & $1 \cdot 149$ & $1 \cdot 159$ & $1-373$ & \\
\hline $\begin{array}{l}P_{c} \text { for } \\
\text { Expt. } 1\end{array}$ & & & & 998.7 & $998 \cdot 8$ & 993.6 & $984 \cdot 6$ & $985 \cdot 1$ & \\
\hline
\end{tabular}

$v=0$ means no initial $v$-filed, $v=v_{g}$ the initial $v$-field is the gradient wind $v$-field. DI stands for dynamic initialization. $24 \mathrm{~h}$ and $48 \mathrm{~h}$ indicate that the DI was performed for 24 and $48 \mathrm{~h}$. $f f=$ standard deviation.

The next interesting question is: What happens if we start with one of the initial temperature structures, say of the control experiment, randomly perturbed. To answer this question the following experiments were performed.

\section{Experiments $4 a, 4 b$ and $4 c$ (figure 15)}

A random temperature perturbation of 0 to $+0 \cdot 1^{\circ} \mathrm{C}, 0$ to $+0 \cdot 4^{\circ} \mathrm{C}$ and 0 to $-0.4^{\circ} \mathrm{C}$ was introduced on the initial control state (figure 3), for experiments, $4 \mathrm{a}, 4 \mathrm{~b}$ and $4 \mathrm{c}$ respectively. Dynamic initialization for $48 \mathrm{~h}$ with an initial state of rest was performed in each case. The results are shown in figure 15 and table 2 . In experiments $4 \mathrm{~b}$ and $4 \mathrm{c}$ the maximum magnitude of the random perturbation was of the same order as that of the maximum magnitude of the initial perturbation. In experiments $4 \mathrm{a}$ and $4 \mathrm{~b}$ the initial vortex was warmer than in the control experiment. In experiment $4 \mathrm{c}$ it was colder.

The results show the following: In experiment $4 a$, for a small random perturbation, the deepening was comparable to experiment $2 \mathrm{a}$, as it should be. Comparing the results of experiments $4 \mathrm{~b}$ and $4 \mathrm{c}$ with those of $4 \mathrm{a}$ or $2 \mathrm{a}$, we notice that the difference in central pressure was of the order $2-3 \mathrm{mb}$ till the end of the experiment.

In table 2 we give the comparative central pressures for all experiments, at different times, along with variances of the deviations in initial conditions of $u, v$ and 6 from the control experiment. We infer the following:

(i) In all the experiments la to $3 b$, the depression deepens compared to the control 
experiment within $24 \mathrm{~h}$ and there was a converging initial radial wind due to a warm centre and dynamical initialization. Thus, these results show that the formation of the tropical cyclone was sensitive to the initial convergence field. After $48 \mathrm{~h}$ the results show that all experiments approached the results of the control experiment.

(ii) The total number of dynamical initialization experiments in table 2 is 9 . Hence the number of degrees of freedom is 8 . At $5 \%$ significant level the statistical $F$ value is $4 \cdot 43$, the $F^{1 / 2}$ approximately being equal to 2 . We can say from table 2 that at $12 \mathrm{~h}$ range the standard deviation of the pressure anomaly (Ap) could be as large as $2 \sigma=2 \times 2.977 \sim 6 \mathrm{mb}$. At 24,36 and $48 \mathrm{~h}$ it could be approximately $2 \sigma=2 \times 1=2 \mathrm{mb}$. We can thus conclude that even with wind and temperature data uncertainties within present-day observational inaccuracies, we can have an error of $6 \mathrm{mb}$ at 12 hours and $2 \mathrm{mb}$ at $24 \mathrm{~h}$ or beyond in the prediction of central pressure. It thus appears that it will not be possible to predict the central pressure to an accuracy of $6 \mathrm{mb}$ up to $12 \mathrm{~h}$ and up to $2 \mathrm{mb}$ at other ranges beyond $24 \mathrm{~h}$.

(iii) The final state after $48 \mathrm{~h}$ was insensitive to small perturbations in the initial state. Thus, the cyclone at some stage of its development behaved like a strongly forced system. In this case, the forcing was internal heating by precipitation. The final state was like an attractor. In view of earlier observations, this suggests that prediction for $12 \mathrm{~h}$ ahead was more difficult than for 24 or $48 \mathrm{~h}$. We are at present examining this aspect with less restrictive model for $h_{M}$.

\section{Discussion and conclusions}

We make the following inferences:

(i) In July, over the head Bay of Bengal, a finite amplitude perturbation of $200 \mathrm{~km}$ radius with a temperature perturbation of $0.4^{\circ} \mathrm{C}$ at the centre, whether it be a warm core, or a warm core with a cold core aloft, can intensify within the framework of a symmetric model. This conclusion will need modification if we consider a threedimensional model.

(ii) The evolution of a cyclone was very sensitive to the initial convergence field. Consequently, a good scheme for initialization is necessary for tropical cyclone prediction,

(iii) It was impossible to predict the central pressure with an accuracy of $6 \mathrm{mb}$ up to $12 \mathrm{~h}$ range and of $2 \mathrm{mb}$ at other ranges beyond with the existing observational accuracies.

(iv) It is difficult at this stage to ascertain the precise dynamical mechanisms that force systems to approach the neighbourhood of a single final state. We feel it is the internal forcing by the release of latent heat that becomes dominant at some point before the mature stage of a cyclone. The initial agencies that trigger the genesis of a cyclone seem to become unimportant as the low pressure develops, and the inflowing air becomes convergent. Consequently, we expect that a large variety of initial conditions could lead to the neighbourhood of the same final state. These experiments show that in predicting the central pressure to an accuracy of, say $2 \mathrm{mb}$ after $48 \mathrm{~h}$, it is possible to do so with diverse initial conditions which differ in general structure. 
Acknowledgements

We thank the Department of Science and Technology for sponsoring this work through the scheme No. 12(14)/84-STP-II. We thank Dr Wada for giving us an opportunity to use her model for these studies. We also thank Dr D V Bhaskar Rao of Andhra University, for continuous interaction during this joint project. One of the authors (PG) thanks CSIR for a post-doctoral fellowship to carry out this work.

\section{Appendix 1. Simplified convective parameterization}

In the (AS) parameterization clouds are characterized by the state of entrainment parameter $\lambda$.

The total cloud mass flux $M_{c}(z)$ at any level is the sum of the individual cloud mass fluxes by clouds detraining at that level or above. Thus we write

$$
M_{c}(z)=\int_{0}^{j_{p}(z)} m_{c}(z, \lambda) \mathrm{d} \lambda=\int_{0}^{\lambda_{p}(z)} m_{B}(\lambda) \eta(z, \lambda) \mathrm{d} \lambda .
$$

Here $\eta(z, \lambda)$ is given by

$$
\begin{array}{cc}
\eta(z, \mathrm{~A})=\exp \left(\lambda\left(z-Z_{B}\right)\right. & \left.\left.Z_{B} \leqslant z \leqslant Z_{D}(\lambda)\right)\right\} \\
\Omega & z_{z_{\nu}}(\lambda)
\end{array}
$$

Thus the cloud mass flux for a particular $\lambda$-type cloud given by $M_{C}(z, \lambda)$ is zero above the level of its detrainment $Z_{D}(\lambda)$. The $A(\lambda)$ type cloud totally detrains at $Z_{D}(\lambda)$. The detrainment $D(z)$ is given by

$$
D(z)=m_{B}\left(\lambda_{D}(z)\right) \cdot \eta\left(z, \lambda_{D}(z)\right)\left[-\mathrm{d} \lambda_{D}(z) / \mathrm{d} z\right] .
$$

The moist-static-energy $h_{c}$ and the total water content in the cloud are obtained by applying the budget equations in one dimension, if the large scale fields are given. The equations used are

$$
\begin{aligned}
& h_{c}(z, \lambda)=1 / \eta(z, \lambda)\left[h_{M}+\lambda \int_{Z_{\mathrm{B}}}^{z} \eta\left(z^{\prime}, \lambda\right) h\left(z^{\prime}\right) \mathrm{d} z^{\prime}\right], \\
& \partial / \partial z\left[\eta(z, \lambda)\left(l_{c}(z, \lambda)+\chi_{c}(z, \lambda)\right)\right]=\lambda \eta(z, \lambda) \chi(z)-\eta(z, \lambda) l_{c}(z, \lambda) \cdot C_{D} .
\end{aligned}
$$

We took $C_{D}$ to be $0 \cdot 002$. The cloud parameters $s_{c}, \chi_{c}$ and $l_{c}$ are estimated for each type by assuming saturation inside clouds. The level of detrainment $Z_{D}(\lambda)$ was obtained by a vanishing buoyancy condition for each 1 type cloud. The equations used for this are

$$
\begin{aligned}
& \chi_{c}(z, \lambda)=\bar{\chi}^{*}(z)+[(\gamma / 1+\gamma) L]\left[h_{c}(z, \lambda)-\bar{h}^{*}\right]_{v} \\
& S_{c}(z, \lambda)=5+\left[h_{c}(z, 1)-\bar{h}^{*}\right] /(1+\mathrm{r}), \\
& S_{v c}\left(z, \lambda_{D}\left(Z_{D}\right)\right)=\bar{S}_{v}(z)
\end{aligned}
$$


where

$$
S_{v}=C_{P}(T+0.608 \chi T)+g z .
$$

Thus $\eta(z, \lambda), s_{c}(z, \lambda), x_{c}(z, \lambda), l(z, \lambda)$ and $\lambda_{D}(z)$ were determined by large scale static control. $m_{B}(\lambda)$ was determined by large-scale dynamic control. For this the closure condition on the cloud work function defined by

$$
A(\lambda)=\int_{z_{\mathrm{B}}}^{z_{p}(\dot{\lambda})} g / C_{p} \bar{T} \eta(z, \lambda)\left[S_{v c}(z, \lambda)-S_{v}(z)\right] \mathrm{d} z
$$

$m_{B}(\hat{\lambda})$ satisfied the conditions

$$
\begin{array}{ll}
m_{B}(\lambda)>0 & \text { if } \partial A(\lambda) / \partial t=0 \\
m_{B}(\lambda)=0 & \text { if } \partial A(\lambda) / \partial t<0 .
\end{array}
$$

In this model three types of clouds were considered. All clouds originate in the mixed layer with bases at the respective lifting condensation level. Type $i=1$ corresponds to clouds that reach the highest level ( $H$ type), $i=2$ refers to clouds (M type) that reach the middle level and $i=3$ ( $L$ type) represents low clouds. After the calculation of $A\left(\lambda_{i}\right)$ for each cloud, $m_{B}\left(\lambda_{i}\right)$ for clouds was determined by a matrix equation of the type

$$
\left|\begin{array}{l}
\partial A\left(\lambda_{1}\right) / \partial t \\
\partial A\left(\lambda_{2}\right) / \partial t \\
\partial A\left(\lambda_{3}\right) / \partial t
\end{array}\right|=|K|\left|\begin{array}{l}
m_{B}\left(\lambda_{1}\right) \\
m_{B}\left(\lambda_{2}\right) \\
m_{B}\left(\lambda_{3}\right)
\end{array}\right|+\left|F_{c}\right|\left|\begin{array}{l}
(\partial \theta / \partial t)_{\mathrm{LS}} \\
(\partial \chi / \partial t)_{\mathrm{LS}}
\end{array}\right|
$$

where subscript LS stands for large scale and the kernels $K$ and $F_{c}$ are determined by $\overline{8}$ and $\chi$ at time step $\tau$ (Wada 1979). The simplifications by Wada were:

(i) Contribution of cloud top level change for $\partial A(\lambda) / \partial \mathrm{tw}$ as neglected.

(ii) Contribution of $\partial Z_{B} / \partial t$ for $\partial A(\lambda) / \lambda t$ was neglected, and

(iii) $h_{M}$ had the upper limit of $\overline{t i}$.

\section{Appendix 2. Notation}

$B \quad$ a suffix indicating the top of the mixed layer

$C \quad$ larger scale condensation per unit time and per unit mass

$C_{D} \quad$ drag coefficient $=2 \cdot 0 \times 10^{-3}$

$C_{\mathrm{P}} \quad$ specific heat of dry air at constant pressure

$D \quad$ suffix indicating detrainment level

$D(z) \quad$ total detrainment of cloud mass flux at level $\mathrm{z}$

$F_{S}, F_{z} \quad$ Air sea exchange of sensible heat and water vapour respectively from the surface to the atmosphere

I Coriolis parameter

$g \quad$ acceleration due to gravity

$h \quad$ moist static energy

$h_{M} \quad$ moist static energy of the mixed layer

$h_{s} \quad$ moist static energy at the surface 
$K_{F}, K_{\chi} \quad$ horizontal eddy coefficients of viscosity and diffusivity $=10^{3} \mathrm{~m}^{2} / \mathrm{s}$

$K_{V} \quad$ vertical eddy coefficient of viscosity $=10 \mathrm{~m}^{2} / \mathrm{s}$

$L \quad$ laten heat of condensation of water vapour

$l \quad$ mixing ratio of liquid water

$M \quad$ suffix indicating mixed layer

$M_{c}(z) \quad$ total vertical cloud mass flux at level $z$

.$P$ pressure

$P_{0} \quad$ reference pressure $=1000 \mathrm{mb}$

$q \quad$ humidity

$R \quad$ gas constant of air

$r \quad$ suffix indicating radical direction

$S \quad$ dry static energy

$T_{M} \quad$ temperature of the mixed layer

$T_{S} \quad$ surface temperature

$t \quad$ time

$v_{r} \quad$ radial wind $\mathrm{m} / \mathrm{s}$

$w \quad$ vertical velocity

$X \quad$ mixing ratio of water vapour

$Z_{B} \quad$ height of the mixed layer top

$\delta_{1}, \delta_{2} \quad$ multipliers to keep or suppress the vertical flow (value $=0$ or 1 )

$f_{1}(P) \quad\left(P / P_{0}\right)^{\kappa}$

$y \quad \mathrm{~L}(\partial q / \partial s)_{p}$

$K \quad\left(R / C_{P}\right)$

$K g \quad$ horizontal eddy coefficient of conductivity $=10^{3} \mathrm{~m}^{2} / \mathrm{s}$

$4 \quad$ liquid water content of the cloud

A entrainment rate of the $\lambda$-type cloud $=$ constant with height

$\lambda_{D}(z) \quad$ A of a cloud detraining at level $z$

$m_{B}(\lambda) \quad$ base mass flux of an individual A cloud

$m_{c}(z, \lambda)$ mass flux in an individual A cloud

$\phi \quad$ a suffix indicating tangential direction

$p \quad$ density of air

$\rho_{s} \quad$ density of air at the surface

$S_{c} \quad$ dry static energy of the cloud

$S_{V C} \quad$ virtual static energy in the cloud

9 potential temperature and also 6 coordinate direction.

$\tau_{r s} \quad$ radial stress at the surface

$\tau_{\theta}, \tau_{r} \quad$ small scale vertical turbulent stress in the 9 (tangential) and $r$ (radial) directions respectively

$\tau_{\theta s} \quad$ tangential stress at the surface

$v_{s} \quad$ magnitude of the surface wind

$v_{r s} \quad$ radial component of the surface wind

$v_{\theta} \quad$ tangential wind $\mathrm{m} / \mathrm{s}$

$v_{\theta s} \quad$ tangential component of the surface wind

$W_{T}(z) \quad$ weights for initial temperature perturbation at level $\mathrm{z}$

$\gamma_{w n} \quad$ humidity mixing ratio of the mixed layer

$\chi_{v_{s}} \quad$ surface humidity mixing ratio

$\nabla_{1}^{2}=\partial^{2} / \partial r^{2}+1 / r 8 / 8 r$ 


\section{References}

Anthes R A, Rosenthal S L and Trout J W 1971 Preliminary results from an asymmetric model of the tropical cyclone; Mon. Weath. Rev. 99 744-758

Arakawa A and Schubert W H 1974 Interaction of a cumulus cloud ensemble with the large scale environment, Part I; J. Atmos. Sci. 31 674-701

Baik J-J, Demaria M and Sethuraman 1990 Tropical cyclone simulations with Betts convective adjustment scheme, Part II, sensitivity experiments; Mon. Weath. Rev. 118 529-541

Charney J and Eliassen A 1964 On the growth of the hurricane depression; J. Atmos. Sci. 21 68-75

Emanuel K A 1986 An air sea interaction theory for tropical cyclones, Part I; J. Atmos. Sci. 43 585-604

Emanuel K A 1989 The finite amplitude nature of tropical cyclogenesis; J. Atmos. Sci. 46 3431-3456

Jordan C L 1958 Mean soundings for the West Indies area; J. Meteorol. 15 91-97

Kurihara Y 1975 Budget analysis of a tropical cyclone simulated in an axisymmetric numerical model; $J$. Atmos. Sci. 32 25-59

Kurihara Y and Tuleya R E 1974 Structure of a tropical cyclone developed in a three-dimensional numerical simulation model; J. Atmos. Sci. 31 893-919

Ooyama K 1964 A dynamical model for the study of tropical cyclone development; Geofis. Int. (Mexico) 4 187-198

Ooyama K 1969 Numerical simulation of the life cycle of tropical cyclones; J. Atmos. Sci. 26 3-40

Rosenthal S 1970 A circularly symmetric primitive equation model of tropical cyclone development containing an explicit water vapor cycle; Mon. Weath. Rev. 98 643-663

Rosenthal S 1971 The response of a tropical cyclone model to variations in boundary layer parameters, initial conditions, lateral boundary conditions, and domain size; Mon. Weath. Rev. 99 767-777. 1

Rosenthal S 1973 Hurricane modelling experiments with a new parameterization for cumulus convection; NOAA Tech. Memorandum ERL WMPO-4

Rotunno R and Emanuel K A 1987 An air sea interaction theory for tropical cyclones, Part II; J. Atmos. Sci. 44 542-561

Shukla J 1981 Dynamical predictability of monthly means; J. Atmos. Sci. 38 2547-2572

Shukla J 1985 Predictability; Adv. Geophys. B28 87-132

Wada M 1977 The properties of typhoon-scale disturbances produced by Arakawa-Schubert parametrization; J. Meteorol. Soc. Jpn. 55 364-391

Wada M 1979 Numerical experiments of the tropical cyclone by use of the Arakawa-Schubert parameterization; J. Meteorol. Soc. Jpn. 57 505-530

Yamasaki M 1968a Numerical simulation of tropical cyclone development with the use of primitive equations; J. Meteorol. Soc. Jpn. 46 178-201

Yamasaki M 1968b A tropical cyclone model with parameterized vertical partitions of released latent heat; J. Meteorol. Soc. Jpn. 46 202-214

Yamasaki M 1968c Detailed analysis of a tropical cyclone simulated with a 13-layer model; Pap. Met. Geophys. 19 559-585

Yamasaki M 1977a A preliminary experiment of the tropical cyclone without parameterizing the effects of cumulus convection; J. Meteorol. Soc. Jpn, 55 11-31

Yamasaki M 1977b The role of surface friction in tropical cyclones; J. Meteorol. Soc. Jpn. 55 559-571 